\title{
Structural dynamics of a plant NLR resistosome: transition from autoinhibition to activation
}

\author{
Jing Wang ${ }^{1}$, Mawsheng Chern ${ }^{2} \&$ Xuewei Chen ${ }^{1 *}$ \\ ${ }^{1}$ State Key Laboratory of Hybrid Rice, Key Laboratory of Major Crop Diseases and Collaborative Innovation Center for Hybrid Rice in \\ Yangtze River Basin, Rice Research Institute, Sichuan Agricultural University at Wenjiang, Chengdu, Sichuan 611130, China; \\ ${ }^{2}$ Department of Plant Pathology, University of California, Davis, CA 95616, USA
}

Received April 6, 2019; accepted April 9, 2019; published online April 12, 2019

Citation: Wang, J., Chern, M., and Chen, X. (2020). Structural dynamics of a plant NLR resistosome: transition from autoinhibition to activation. Sci China Life Sci 63, 617-619. https://doi.org/10.1007/s11427-019-9536-x

Despite the lack of a cellular, adaptive immune system, plants share with animals an innate immune system and can effectively fight off pathogen attack using two layers of defense response: the first layer consists of plasma membrane-localized pattern recognition receptors (PRRs) that recognize pathogen-associated molecular patterns (PAMPs) and can mount a pattern-triggered immunity (PTI); the second layer comprises cytoplasmic nucleotide-binding, leucine-rich repeats receptors (NLRs) that recognize pathogensecreted effectors and mount an effector-triggered immunity (ETI) (Shamrai, 2014). Plants contain hundreds of NLR genes constituting one of the largest families of genes. Because plant $N L R$ genes can mount the strongest immune response, often including a hypersensitive response, upon recognition of its cognate effector, they were initially characterized as resistance $(R)$ genes and conform to the genefor-gene hypothesis postulated in 1955 (Hh, 1955; McHale et al., 2006). The plant NLR-mediated ETI response is thus comparable to the animal inflammatory response.

NLR proteins contain a nucleotide-binding oligomerization domain (NOD), a C-terminal leucine-rich repeat (LRR) domain, and a variable N-terminal domain (Wu et al., 2017). NLR proteins are also present in animals sharing a similar domain architecture, including NOD and LRR domains. However, in contrast to plant NLRs which detect race-spe-

*Corresponding author (email: xwchen88@163.com) cific pathogen effectors, animal NLRs detect PAMPs and danger-associated molecular patterns (DAMP) that are widely present and non-race specific, suggesting a potential fundamental difference between the NLR-mediated immune responses in plants and animals (Bentham et al., 2017). Although much important progress has been made in the study of plant NLRs, many pivotal questions remain unanswered, such as how are NLR proteins activated structurally? Despite the importance and high degree of conservation of plant NLR proteins, it remains a great challenge to purify and crystalize NLR proteins in order to solve their structure.

In two recent Science articles (Wang et al., 2019b; Wang et al., 2019a), Chai's, Zhou's and Wang's groups reported exciting progress in tackling this important issue. Previously, these groups have spent many years investigating structures and molecular mechanisms of plant immune receptors and have made great contributions. For example, in 2013, Chai's and Zhou's groups solved the crystal structure of FLS2, a representative of the large family of plant PRRs, providing great insights into the activation of a plant PRR complex that confers a PTI response (Sun et al., 2013). In the recent articles, they crystalized the NLR immune receptor ZAR1 (Hopz-Activated Resistance 1) complex, which confers an ETI response, and observed a structural change that explains the mechanism underlying the switch from autoinhibition to activation (Figure 1). Arabidopsis ZAR1 is a canonical coiled-coil (CC)-type NLR protein which often works to- 


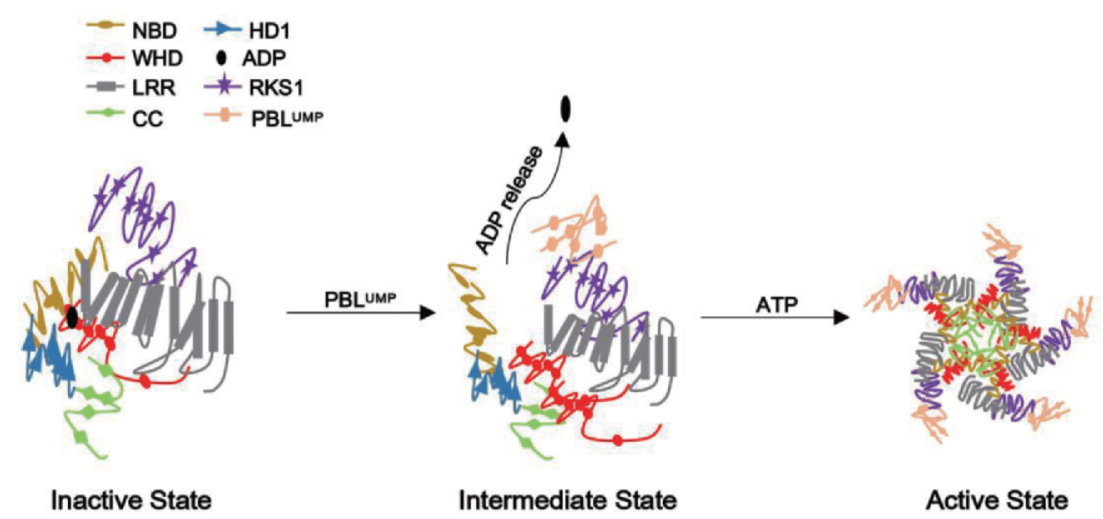

Figure 1 (Color online) Structural dynamics of ZAR1: transition from autoinhibition to activation. Commonly, ZAR1-RKS1 stays in an inactive state maintained by the autoinhibition of ZAR1. After PBL2 becomes uridylylated by effector AvrAC, PBL2UMP emerges as a ligand to initiate the activation of ZAR1. PBL2UMP is recruited by ZAR1-RKS1 and the interaction between PBL2UMP and RKS1 promotes the release of ADP from ZAR1, moving ZAR1 into an intermediate state. Binding of an ATP to the complex further induces structural remodeling of the ZAR1 complex, triggering the formation of a pentameric ZAR1-RKS1-PBL2UMP complex, which is an active state of ZAR1.

gether with other proteins to form a NLR complex recognizing type III secreted effectors (Lewis et al., 2010; Wang et al., 2015; Seto et al., 2017). Previous study showed that ZAR1 associates with RKS1 to form a complex; this complex exclusively recruits PBL2 only when PBL2 is uridylylated by effector AvrAC secreted by Xanthomonas campestris pv. campestris. Uridylylated PBL2 (PBL2UMP) acts as a ligand to activate the ZAR1-RKS1 complex to initiate an ETI response (Wang et al., 2015).

In the two recent Science articles, the authors first investigated the 3D structure of the ZAR1-RKS1 complex in the monomeric form, which represents the inactive state of ZAR1. Based on a 3D analysis, ZAR1 contains five structural domains: ZAR1NBD, ZAR1HD1, ZAR1WHD, ZAR1LRR and ZAR1CC. The relative positions of these domains are important to the autoinhibition of ZAR1. In the inactive state, ZAR1LRR blocks the dorsal-ventral stacking of ZAR1NBD keeping ZAR1 in the monomeric form; the ZAR1WHD domain keeps an ADP in a pocket excluding binding of an ATP, contributing to the inhibition of ZAR1 activation; the intramolecular interactions among these domains cooperatively stabilize the inactive conformation of ZAR1. Furthermore, the authors built a model of ZAR1RKS1-PBL2UMP in the monomeric state, providing evidence supporting a direct interaction between PBL2UMP and RKS1. Interestingly, the recognition of PBL2UMP triggers the formation of a ZAR1-RKS1- PBL2UMP intermediate complex by stabilizing the segment of RKS1, leading to the replacement of the ADP molecule by an ATP molecule associated with ZAR1 (Wang et al., 2019b).

More strikingly, the binding of an ATP/dATP to the complex induces oligomerization of the ZAR1-RKS1PBL2UMP complex in its tertiary structure and stabilizes the active conformation of ZAR1. This active ZAR1 complex, called ZAR1 resistosome, contains five molecules of the
ZAR1-RKS1-PBL2UMP complex, forming a wheel-like pentamer maintained by the association among the five ZAR1 domains. During activation, the structural remodeling and fold switching of ZAR1 happens, releasing the Nterminal amphipathic $\alpha$-helix of ZAR1, which is required for plasma membrane association and the immunity conferred by ZAR1 (Wang et al., 2019a). Thus, by combining crystalstructural, biochemical and genetic analyses, these researchers have resolved the molecular transition from autoinhibition to activation of ZAR1, providing an excellent model for plant NLRs that is different from animal models. The discovery of this plant resistosome is unprecedented and will have far-reaching impacts. The newly discovered resistosome is even considered a plant inflammasome by experts in the scientific field (Dangl and Jones, 2019).

Emerging evidence suggests that activation of plant NLRs includes direct and indirect interactions between receptors and effectors; ZAR1 recognizes its cognate effector indirectly (Dodds and Rathjen, 2010, Wang et al., 2015). Whether those NLRs activated by direct interaction use a similar model as ZAR1 remains to be elucidated. In addition, while NLRs play an essential role in plant immunity, they often inhibit plant growth and reduce yields (Tian et al., 2003; MacQueen et al., 2016; Deng et al., 2017; Chakraborty et al., 2018). Fortunately, plants have evolved several ways to tightly control the activity of NLRs (Richard et al., 2018). These two Science articles have discovered the molecular mechanism that ensures ZAR1 autoinhibition in the absence of the pathogen and activation upon pathogen infection. They identified several amino acid residues required for maintaining the correct ZAR1 conformation to inhibit activation and for the transition to activation. This presents an intriguing new opportunity to engineer NLR proteins for desired disease resistance with reduced fitness cost. 
Compliance and ethics The author(s) declare that they have no conflict of interest.

\section{References}

Bentham, A., Burdett, H., Anderson, P.A., Williams, S.J., and Kobe, B. (2016). Animal NLRs provide structural insights into plant NLR function. Ann Bot 5, mew171.

Chakraborty, J., Ghosh, P., and Das, S. (2018). Autoimmunity in plants. Planta 248, 751-767.

Dangl, J.L., and Jones, J.D.G. (2019). A pentangular plant inflammasome. Science 364, 31-32.

Deng, Y., Zhai, K., Xie, Z., Yang, D., Zhu, X., Liu, J., Wang, X., Qin, P., Yang, Y., Zhang, G., et al. (2017). Epigenetic regulation of antagonistic receptors confers rice blast resistance with yield balance. Science 355 , 962-965.

Dodds, P.N., and Rathjen, J.P. (2010). Plant immunity: towards an integrated view of plant-pathogen interactions. Nat Rev Genet 11, 539-548.

Hh, F. (1955) Host-parasite interaction in flax rust-its genetics and other implications. Phytopathology 45, 680-685.

Lewis, J.D., Wu, R., Guttman, D.S., and Desveaux, D. (2010). Allelespecific virulence attenuation of the Pseudomonas syringae HopZ1a type III effector via the Arabidopsis ZAR1 resistance protein. PLoS Genet 6, e1000894.

MacQueen, A., Sun, X., and Bergelson, J. (2016). Genetic architecture and pleiotropy shape costs of Rps2-mediated resistance in Arabidopsis thaliana. Nat Plants 2, 16110.

McHale, L., Tan, X., Koehl, P., and Michelmore, R.W. (2006). Plant NBS-
LRR proteins: adaptable guards.. Genome Biol 7, 212

Richard, M.M.S., Gratias, A., Meyers, B.C., and Geffroy, V. (2018). Molecular mechanisms that limit the costs of NLR-mediated resistance in plants. Mol Plant Pathol 19, 2516-2523.

Seto, D., Koulena, N., Lo, T., Menna, A., Guttman, D.S., and Desveaux, D. (2017). Expanded type III effector recognition by the ZAR1 NLR protein using ZED1-related kinases. Nat Plants 3, 17027.

Shamrai, S.N. (2014). Plant immune system: the basal immunity. Tsitol Genet 48, 67-82.

Sun, Y., Li, L., Macho, A.P., Han, Z., Hu, Z., Zipfel, C., Zhou, J.M., and Chai, J. (2013). Structural basis for flg22-induced activation of the Arabidopsis FLS2-BAK1 immune complex. Science 342, 624-628.

Tian, D., Traw, M.B., Chen, J.Q., Kreitman, M., and Bergelson, J. (2003). Fitness costs of R-gene-mediated resistance in Arabidopsis thaliana. Nature 423, 74-77.

Wang, G., Roux, B., Feng, F., Guy, E., Li, L., Li, N., Zhang, X., Lautier, M., Jardinaud, M.F., Chabannes, M., et al. (2015). The decoy substrate of a pathogen effector and a pseudokinase specify pathogen-induced modified-self recognition and immunity in plants. Cell Host Microbe $18,285-295$.

Wang, J., Hu, M., Wang, J., Qi, J., Han, Z., Wang, G., Qi, Y., Wang, H.W., Zhou, J.M., and Chai, J. (2019a). Reconstitution and structure of a plant NLR resistosome conferring immunity. Science 364, eaav5870.

Wang, J., Wang, J., Hu, M., Wu, S., Qi, J., Wang, G., Han, Z., Qi, Y., Gao, N., Wang, H.W., et al. (2019b). Ligand-triggered allosteric ADP release primes a plant NLR complex. Science 364 , eaav5868.

Wu, C.H., Abd-El-Haliem, A., Bozkurt, T.O., Belhaj, K., Terauchi, R., Vossen, J.H., and Kamoun, S. (2017). NLR network mediates immunity to diverse plant pathogens. Proc Natl Acad Sci USA 114, 8113-8118. 